\title{
The \\ Geometric Process and Its Applications
}


This page intentionally left blank 


\section{The \\ Geometric Process and Its Applications}

\section{Yeh Lam}

The University of Hong Kong \& Northeastern University at Qinhuangdao, China 


\section{Published by}

World Scientific Publishing Co. Pte. Ltd.

5 Toh Tuck Link, Singapore 596224

USA office: 27 Warren Street, Suite 401-402, Hackensack, NJ 07601

UK office: 57 Shelton Street, Covent Garden, London WC2H 9HE

\section{British Library Cataloguing-in-Publication Data}

A catalogue record for this book is available from the British Library.

\section{THE GEOMETRIC PROCESS AND ITS APPLICATIONS}

Copyright () 2007 by World Scientific Publishing Co. Pte. Ltd.

All rights reserved. This book, or parts thereof, may not be reproduced in any form or by any means, electronic or mechanical, including photocopying, recording or any information storage and retrieval system now known or to be invented, without written permission from the Publisher.

For photocopying of material in this volume, please pay a copying fee through the Copyright Clearance Center, Inc., 222 Rosewood Drive, Danvers, MA 01923, USA. In this case permission to photocopy is not required from the publisher.

ISBN-13 978-981-270-003-2

ISBN-10 981-270-003-X

Printed in Singapore. 


\section{To my parents: \\ Kai Cheung Lam and Lai Ying Yip}


This page intentionally left blank 


\section{Preface}

The motivation of introducing geometric process is due to many practical problems in different fields, including science, engineering, medicine and social science. In maintenance problems, as most systems are deteriorating, the successive operating times of a system after repair will be decreasing, while the consecutive repair times after failure will be increasing. In epidemiology study, the number of infected cases usually shows an increasing trend in the early stage, and is stable in the middle stage, but has a decreasing trend in the late stage. In economics research, the economic development of a country or a region often shows a periodic cycle, so that its gross national product (GNP) will be increasing in the early stage of a cycle, and stable in the middle stage of the cycle, then decreasing in the late stage of the cycle. Many models such as minimal repair model, nonlinear regression model, nonlinear time series model, were developed for treating problems of the above phenomena with trend. However, it seems that a more direct approach is to introduce a monotone process model. As a simple monotone process, Lam (1988a, b) first introduced the geometric process.

Definition A stochastic process $\left\{X_{i}, i=1,2, \ldots\right\}$ is called a geometric process if there exists a real $a>0$ such that $\left\{a^{i-1} X_{i}, i=1,2, \ldots\right\}$ form a renewal process. The real number $a$ is called the ratio of the geometric process.

Clearly, a geometric process is stochastically decreasing, if $a \geq 1$; it is stochastically increasing, if $0<a \leq 1$. It will become a renewal process, if $a=1$. Therefore, the geometric process is a generalization of a renewal process.

So far, much research work has been done in developing the theory and applications of geometric process. Many fruitful theoretical results and in- 
teresting applications have attracted more and more attention. As a result, the second edition of Encyclopedia of Statistical Science has added "Geometric Process" (see Lam (2006) for reference) as one new item.

In this book "Geometric Process and Its Application", we shall summarize the research work on theory and applications of geometric process developed since 1988. We expect that the publication of this book will stimulate further research in this topic and strengthen the practical applications. We sincerely hope more new results will follow after the publication of this book. We also hope the penetration of geometric process into different application fields will be accelerated after this book comes out.

Chapter 1 is a chapter of preliminaries. It contains a brief review of the Poisson process and renewal process. Stochastic order and some classes of lifetime distribution are then studied. In addition, a new class of lifetime distribution, namely earlier preventive repair better (worse) than later in expectation, is introduced. In Chapter 1, some martingale theory is reviewed. Furthermore, a formula for the rate of occurrence of failure is also studied.

In Chapter 2, the definition of geometric process is introduced, followed by the study of its probability properties.

In Chapter 3, like the renewal function in a renewal process, the expected number of events occurred by time $t$ in a geometric process is defined as the geometric function of the geometric process. As the renewal function, the geometric function is also very important in application such as in warranty problem and block replacement model. In Chapter 3, several methods for the evaluation of the geometric function are suggested, these methods are then compared with a simulation method.

In Chapter 4, the statistical inference of a geometric process is studied. To do this, two statistics are introduced for testing if a data set agrees with a geometric process. Afterward, some nonparametric and parametric estimators for the parameters in a geometric process are proposed. The asymptotic normality of these estimators are then studied.

In Chapter 5, a geometric process model is applied to analysis of data with trend. Many real data sets are analyzed. In comparison with the results obtained by other three models, the geometric process model is the best model among four models. Furthermore, a threshold geometric process model is introduced for analyzing data with multiple trends. As an example, the threshold geometric process model is used to study the data sets of daily infected cases in SARS. 
In Chapter 6, the application of geometric process to the maintenance problem of a one-component system is studied. The optimal replacement policy is determined analytically. Furthermore, the monotonicity of the optimal replacement policy is discussed. Afterward, a monotone process model for a multiple state system is considered, and a threshold geometric process maintenance model is introduced for a system with multiple trends, in particular for a system with a bathtub failure rate function. Moreover, a shock geometric process model and a $\delta$-shock geometric process model are considered, both models study the effect of environment on the system. Then as a preventive repair is very useful in increasing the availability and reliability of a system, at the end of Chapter 6 , a geometric process model with preventive repair is considered.

In Chapter 7, the reliability analysis for a two-component series, parallel and cold-standby systems respectively, is discussed. A geometric process maintenance model for a two-component cold standby system is also investigated.

In Chapter 8, the applications of geometric process to other topics in operational research is discussed. We consider here the applications in queueing theory and warranty problem.

Thus, Chapters 2-4 concentrate on the theoretical research of the probability theory and statistical inference of geometric process. Therefore, Chapters 2-4 form the foundation of the application of the geometric process. Nevertheless, Chapters 5-8 demonstrate the applications of geometric process to statistics, applied probability, operational research and management science.

The prerequisite for reading this book is an undergraduate calculus course plus a probability and statistics course. Only a few sections or results which are marked with an asterisk $*$ may require more advanced mathematics. However, the readers can skip over these parts without affecting the understanding of the main contents in the manuscript.

On the one hand, this book is a reference book for the researchers who are interested in geometric process theory or its application; on the other hand, it is a handbook for practitioners such as maintenance managers or reliability engineers or data analysts. Moreover, this book can be used as a postgraduate textbook or senior undergraduate textbook for statistics, applied probability, operational research, management science and reliability courses for one semester.

I take this opportunity to thank Professors A. C. Atkinson, M. D. 
Cheng, D. R. Cox, S. S. Ding, L. C. Thomas and H. Tong, who were my teachers respectively during my undergraduate and postgraduate studies, for their continuing instruction and advice. I would also like to thank Professor Y. L. Zhang for our long-term cooperation in research, especially in the joint research of geometric process. I am grateful to Dr. Jennifer S. K. Chan and Dr. Yayong Tang for their assistance in preparation of the manuscript. I should express my gratitude to all of my friends and students for their help, concern and encouragement. I should also express my thanks to the colleagues of World Scientific for their effort and work, so that this book can be prosperously published.

Last but not least, I would like to thank my wife Chiu Bing Tan and my sons Fong Lam and Ying Lam for their continuous concern and support in order that I can devote time and energy to teaching, research and preparation of this book.

Yeh Lam

The University of Hong Kong and Northeastern University at Qinhuangdao ylam@saas.hku.hk

May 2007 


\section{Contents}

Preface vii

1. Preliminaries 1

1.1 Introduction . . . . . . . . . . . . . . 1

1.2 The Poisson Process . . . . . . . . . . . . . . . . . 2

1.3 The Renewal Process . . . . . . . . . . . . . . . . 8

1.4 Stochastic Order and Class of Lifetime Distributions . . . 18

$1.5 *$ Martingales . . . . . . . . . . . . . . 26

$1.6 *$ The Rate of Occurrence of Failures . . . . . . . . . . 31

1.7 Notes and References . . . . . . . . . . . . . . . . . 36

2. Geometric Process 37

2.1 Introduction . . . . . . . . . . . . . . . . 37

2.2 Geometric Process . . . . . . . . . . . . . . . . . . 37

2.3 Age, Residual Life and Total Life . . . . . . . . . . . . 42

2.4 Limit Theorems for Geometric Process . . . . . . . . . . 45

2.5 A Geometric Process with Exponential Distribution ... 50

2.6 Notes and References . . . . . . . . . . . . . . . . 53

3. Geometric Function $\quad 55$

3.1 Introduction . . . . . . . . . . . . . . 55

3.2 Geometric Equation . . . . . . . . . . . . . 56

3.3 Existence of Geometric Function . . . . . . . . . . . 57

3.4 General Solution to Geometric Equation . . . . . . . . . . 61

$3.5 *$ Analytic Solution to Geometric Equation . . . . . . . 63

3.6 Numerical Solution to Geometric Equation . . . . . . . 79 
$3.7 \quad$ Approximate Solution to Geometric Equation . . . . . . . 83

3.8 Comparison with Simulation Solution to Geometric Equation 89

3.9 Exponential Distribution Case . . . . . . . . . . . 98

3.10 Notes and References . . . . . . . . . . . . . . . . . . . 100

4. Statistical Inference of Geometric Process 101

4.1 Introduction . . . . . . . . . . . . . . . . . 101

4.2 Hypothesis Testing for Geometric Process . . . . . . . . . 101

4.3 Estimation of Parameters in Geometric Process . . . . . . 104

4.4 Asymptotic Distributions of the Estimators . . . . . . . 106

4.5 Parametric Inference for Geometric Process . . . . . . . . 113

4.6 Notes and References . . . . . . . . . . . . . . . . . . . . 118

5. Application of Geometric Process to Data Analysis 121

5.1 Introduction . . . . . . . . . . . . . . . . . . . . 121

5.2 Data Analysis by Geometric Process Model . . . . . . . . 122

5.3 Data Analysis by Poisson Process Models . . . . . . . . . 123

5.4 Real Data Analysis and Comparison . . . . . . . . . . 125

5.5 Analysis of Data by a Threshold Geometric Process Model 142

5.6 Notes and References . . . . . . . . . . . . . . . . 152

6. Geometric Process Maintenance Model 155

6.1 Introduction . . . . . . . . . . . . . . . . . 155

6.2 A Geometric Process Maintenance Model . . . . . . . . . 156

6.3 Optimal Replacement Policy . . . . . . . . . . . . . . 161

6.4 Monotonicity of the Optimal Policy for a Deteriorating System . . . . . . . . . . . . . . . . . . . 164

6.5 A Monotone Process Model for a Multistate System . . . 168

6.6 A Geometric Process Shock Model . . . . . . . . . . . . . 182

$6.7 \quad$ A Geometric Process $\delta$-Shock Model . . . . . . . . . . . 193

6.8 A Threshold Geometric Process Maintenance Model . . . 201

6.9 A Geometric Process Preventive Maintenance Model . . . 210

6.10 Notes and References . . . . . . . . . . . . . . . . . . . . . 223

7. Application to Analysis of System Reliability 227

7.1 Introduction . . . . . . . . . . . . . . . . 227

7.2 Reliability Analysis for a Series System . . . . . . . . . 227

7.3 Reliability Analysis for a Parallel System . . . . . . . . 234 
7.4 Reliability Analysis for a Cold Standby System . . . . . . 239

7.5 A Geometric Process Maintenance Model for a Cold Standby System . . . . . . . . . . . . . . . . . 249

7.6 Notes and References . . . . . . . . . . . . . . . . . . 252

8. Applications of Geometric Process to Operational Research 255 8.1 Introduction . . . . . . . . . . . . . . . . . 255

8.2 A Geometric Process M/M/1 Queueing Model . . . . . . 255

8.3 A Geometric Process Warranty Model . . . . . . . . . . 274

8.4 Notes and References . . . . . . . . . . . . . . . . . . . 284

Appendix A SARS Data Sets 286

A.1 Hong Kong SARS Daily Infected Case Data . . . . . . . . 286

A.2 Singapore SARS Daily Infected Case Data . . . . . . . . 287

A.3 Ontario SARS Daily Infected Case Data . . . . . . . . . 288

A.4 Taiwan SARS Daily Infected Case Data . . . . . . . . . 289

Bibliography 291

$\begin{array}{ll}\text { Index } & 297\end{array}$ 